University of Wollongong

Research Online

Australian Institute for Innovative Materials -

Papers

Australian Institute for Innovative Materials

2013

Colour tunable electrochromic devices based on PProDOT-(Hx)2 and PProDOT-(EtHx)2 polymers

\author{
Bo Weng \\ University of Wollongong, bweng@uow.edu.au \\ Syed Ashraf \\ University of Wollongong, syed@uow.edu.au \\ Peter C. Innis \\ University of Wollongong, innis@uow.edu.au \\ Gordon G. Wallace \\ University of Wollongong, gwallace@uow.edu.au
}

Follow this and additional works at: https://ro.uow.edu.au/aiimpapers

Part of the Engineering Commons, and the Physical Sciences and Mathematics Commons

Research Online is the open access institutional repository for the University of Wollongong. For further information contact the UOW Library: research-pubs@uow.edu.au 


\title{
Colour tunable electrochromic devices based on PProDOT-(Hx)2 and PProDOT- (EtHx)2 polymers
}

\begin{abstract}
The most commonly used method to tune the colour transition states of an ECD is to modify the chemical structure of the electrochromic polymers to achieve the desired transparent to dark state switching colours. However, this approach can present significant synthetic challenges that typically result in a compromise in device performance parameters such as contrast range or stability as well as solvent processability. In this study we have investigated tuning the dark-state colour of an ECD (at $+0.8 \mathrm{~V})$ by solution mixing poly(3,3-dihexyl-3,4-dihydro-2H-thieno[3,4-b][1,4]dioxepine) (PProDOT-(Hx)2), which has an excellent contrast performance but with an esthetically undesirable purple colour transition, with poly(3,3-bis(2-ethylhexyl)-3,4-dihydro-2H-thieno [3,4-b][1,4]dioxepine) (PProDOT-(EtHx)2), a material with a poorer contrast range but with more esthetic blue colour transition. The influence of mixtures of two cathodically colouring materials, PProDOT-( $\mathrm{Hx}) 2$ and PProDOT- $(\mathrm{EtHx}) 2$, on the spectroelectrochemistry and assembled ECDs was explored. Photopic contrast, electrochemical properties and the influence of the type of ionic liquid electrolyte employed in the device assembly were also investigated to determine how the dark-state colour of ECDs can be tuned while maintaining device contrast over $55 \%$.
\end{abstract}

\section{Keywords}

electrochromic, devices, pprodot, hx, 2, colour, ethx, tunable, polymers

\section{Disciplines}

Engineering | Physical Sciences and Mathematics

\section{Publication Details}

Weng, B., Ashraf, S., Innis, P. C. \& Wallace, G. G. (2013). Colour tunable electrochromic devices based on PProDOT-(Hx)2 and PProDOT-(EtHx)2 polymers. Journal of Materials Chemistry C, 1 (44), 7430-7439. 


\title{
Colour Tunable Electrochromic Devices Based on PProDOT-(Hx) $)_{2}$ and PProDOT-(EtHx) $)_{2}$ Polymers
}

\author{
Bo Weng, Syed Ashraf, Peter C. Innis*, and Gordon G. Wallace \\ Received (in $X X X, X X X)$ Xth $X X X X X X X X X 20 X X$, Accepted Xth $X X X X X X X X X 20 X X$ \\ ${ }_{5}$ DOI: 10.1039/b000000x
}

\begin{abstract}
The most common used method to tune the colour transition states of an ECD is to modify the chemical structure of the electrochromic polymers to achieve the desired transparent to dark state switching colours. However, this approach can present significant synthetic challenges that typically results in a compromise in device performance parameters such as contrast range or stability as well as solvent

10 processability. In this study we have investigated tuning the dark - state colour of an ECD (at $+0.8 \mathrm{~V}$ ) by solution mixing poly(3, 3 - Dihexyl - 3, 4 - dihydro - 2H - thieno [3, 4-b] [1, 4]dioxepine) (PProDOT$(\mathrm{Hx})_{2)}$, which has an excellent contrast performance but with an esthetically undesirable purple colour transition, with poly(3,3 - bis (2 - ethylhexyl) - 3, 4 - dihydro - 2H - thieno [3, 4-b] [1, 4]dioxepine) (PProDOT-(EtHx $)_{2}$, a material with a poorer contrast range but with more esthetic blue colour transition. 15 The influence of mixtures of two cathodically colouring materials, PProDOT-(Hx) $)_{2}$ and PProDOT-

$(\mathrm{EtHx})_{2}$ on the spectroelectrochemistry and assembled ECDs were explored. Photopic contrast, electrochemical properties and the influence of the type of ionic liquid electrolyte employed in the device assembly were also investigated to determine how the dark-state colour of ECDs can be tuned while maintaining device contrast over 55\%.
\end{abstract}

\section{${ }_{20} 1$ Introduction}

Conducting organic electronics ${ }^{1-4}$ is an area of great interest with conducting polymer based devices, such as organic transistors, polymer lighting emitting diodes (PLEDs) ${ }^{5,}$, , organic photovoltaic devices $(\mathrm{OPV})^{7-9}$ and electrochromic devices 25 (ECDs) ${ }^{10-12}$ being realized. Among these, the electrochromic properties of $\pi$ - conjugated polymers has attracted a broad range of attention due to their potential applications in self-dimming car mirrors $^{13,14}$, electrochromic (EC) screens ${ }^{15}$ and EC windows ${ }^{16,17}$ for the aero or space industries.

30 In general, electrochromism can be defined as the process of switching the UV-vis absorption profile of a material with application of an electric field or passing of electronic charge ${ }^{17-19}$. Over the years, a number of EC materials, including metal oxides $^{20,21}$, such as $\mathrm{WO}_{3}$, and conducting polymers, ${ }^{22-24}$ such as

35 polyethylenedioxythiphene (PEDOT), have been reported and reviewed. These studies range from the synthesis of EC materials with high contrast ${ }^{25-27}$ to scalable electrochromic device (ECD) fabrication $^{28,29}$.

Conducting polymers with electrochromic properties have 40 received special attention due to their high contrast ratios, fast switching speed and solution process ability ${ }^{33}$. In addition, the colour of EC polymers can be tuned by synthetic modification of the monomer structure ${ }^{25,34}$. Reynolds et al. first established a method to fabricate dual polymer complementary ECDs by 45 assembling paired dark-state and charge matched cathodically and anodically coloring EC polymers to increase colour efficiency $^{35,36}$. Since then, a series of complementary conducting polymer ECDs have been assembled via spin coating ${ }^{32}$, spray coating $^{33,36}$ and roll to roll printing ${ }^{37}$ to produce devices that

50 bridge the gap between laboratory and industrial application.

While the material properties and fabrication techniques for an ECD have been studied in detail, the ability to tune the colour while maintaining high contrast required for a practical device can provide a roadblock to application. The synthesis of new 55 substituted electrochromics materials can be time consuming, challenging and result in complex processing issues (such as EC solubility) as a result of the modification of the electrochromes molecular structure. To simplify this problem, we have developed a composite material strategy that mixes well characterized 60 (solvent processable) EC polymers, that have distinctly different colours and contrast ranges, at different ratios to create a more esthetically pleasing colour and an improved device contrast than the individual components can achieve by themselves. This study was undertaken using two complementary colouring 65 electrochromic polymers. Poly (3, 3 - Dihexyl - 3, 4 - dihydro $2 \mathrm{H}$ - thieno [3, 4-b] [1, 4]dioxepine) (PProDOT-(Hx) $)_{2}$ ) and $\operatorname{poly}(3,3$ - bis (2 - ethylhexyl) - 3, 4 - dihydro - 2H - thieno [3, 4-b] [1, 4]dioxepine) (PProDOT-(EtHx) $)_{2}$ (Figure 1 (a) and (b)) were selected as cathodically colouring materials due to their 70 reported colours, solubility, process ability and large transmissive difference between dark (reduced) and bleached (oxidized) state ${ }^{25,27,30}$. Poly(2-methoxyaniline-5-sulfonic acid) (PMAS) $^{31}$ (Figure 1 (c)) was selected as anodically colouring 
material due to its high transparency in the bleached (reduced) state. The aim of this study was to design and fabricate a high quality portable $(5 \mathrm{~cm} \times 5 \mathrm{~cm})$ transmissive ECD with colourless to dark colour transitions that can be tuned from purple to blue, 5 with contrast changes above 55\%.

\section{Experimental}

\subsection{Synthesis of PProDOT-(Hx) $)_{2}$, PProDOT-(EtHx $)_{2}$ and} PMAS

Synthesis of Poly(3,3-Dihexyl-3,4-dihydro-2H-thieno[3,4$10 \mathrm{~b}][1,4]$-dioxepine) [PProDOT-(Hx) $\left.{ }_{2}\right]$ and poly(3,3-bis(2ethylhexyl)-3-4-dihydro-2H-thieno[3,4-b][1,4]-dioxepine

[PProDOT-(EtHx $)_{2}$ ] was carried out, following the literature method $\underline{25}$ with some modification. Firstly, 2,2-Dihexylpropane1,3-diol or 2,2-Bis(2-ethylhexyl)-propane-1,3-diol was 15 synthesized by an alkylation of diethyl malonate using sodium hydride and the corresponding alkyl bromide followed by $\mathrm{LiAlH}_{4}$ reduction and further purification by distillation under vacuum. Transetherification of 3,4-Dimethoxy thiophene with the diol produced resulted the corresponding 3,3-Dihexyl-3,4-dihydro20 2H-thieno[3,4-b][1,4]- dioxepine) [ProDOT-(Hx) 2 ] after a single purification by column chromatography with silica gel with $3: 2$ hexane/dichloromethane and 3,3-Bis(2-ethylhexyl)-3,4- dihydro2H-thieno[3,4-b][1,4]-dioxypine [ProDOT-(EtHx) $)_{2}$ ] after a twice purification by column chromatography on silica gel first with 25 3:2 hexane/dichloromethane followed by 3:1 hexane/dichloromethane. Bromination of ProDOT- $(\mathrm{Hx})_{2}$ or ProDOT-(EtHx $)_{2}$ at thethiophene ring resulted in 6,8-Dibromo3,3-dihexyl-3,4-dihydro-2H-thieno[3,4-b][1,4]-dioxepine [ProDOT-(Hx) ${ }_{2} \mathrm{Br}_{2}$ ] or 6,8-Dibromo-3,3-bis(2-ethylhexyl)-3,430 dihydro-2H-thieno[3,4-b][1,4]dioxepine[ProDOT-(EtHx) ${ }_{2} \mathrm{Br}_{2}$ ], after two purifications by column chromatography with $4: 1$ hexane/dichloromethane. The NMR results were in agreement to the literature method ${ }^{25}$.

Chemical polymerization of ProDOT-(Hx) $)_{2} \mathrm{Br}_{2}$ or 35 [ProDOT $(\mathrm{EtHx})_{2} \mathrm{Br}_{2}$ ] was carried out by using Grignard's reagent (methyl magnesium bromide $1 \mathrm{M}$ in THF) in presence of $\mathrm{Ni}(\mathrm{dppp}) \mathrm{Cl}_{2}$ catalyst followed by purification using soxhlet extraction with methanol 24h, hexane $48 \mathrm{~h}$ and chloroform $24 \mathrm{~h}$. Polymerised PProDOTs were also characterized by NMR which 40 was similar to the literature ${ }^{25}$ and its molecular weight determined by GPC against polystyrene relative standards. The moleculare weights were found to be PProDOT-(EtHx) $)_{2} \mathrm{Mn}$ 65,000 Mw 66,000 PDI 1.06; PProDOT-(Hx) 2 Mn $40217 \mathrm{Mw}$ 42000 PDI 1.04 .

45 Poly (2-Methoxy aniline-5-sulfonic acid) (PMAS) was synthesized chemically by the polymerization of $50 \mathrm{~g}(0.2 \mathrm{M})$, 2Methoxy aniline-5-sulfonic acid (MAS) dissolved in 500mL of water (Milli-Q) and adding approximately $15 \mathrm{~mL}$ of $\mathrm{NH}_{3}(28 \%$ $\mathrm{w} / \mathrm{w})$ slowly to $\mathrm{pH} 4$. To this stirred solution $71.4 \mathrm{~g}(0.31 \mathrm{M})$ of 50 ammonium persulfate dissolved in $250 \mathrm{~mL}$ of water was added at one portion as an oxidant at $5-10{ }^{\circ} \mathrm{C}$. The reaction mixture was stirred overnight at the same temperature to complete the reaction. The resultant polymer in solution was purified by the

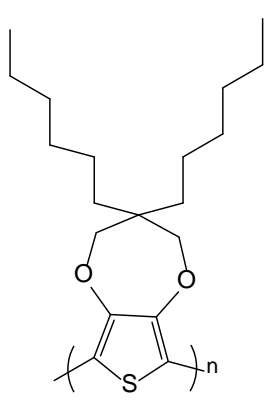

(a)

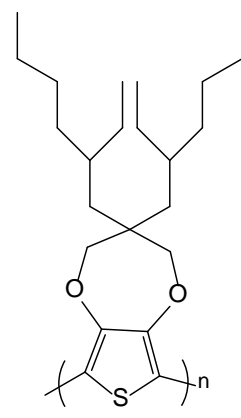

(b)

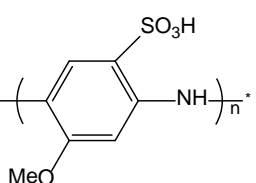

(c)
Fig. 1 Chemical structure of (a) PProDOT-(Hx) $)_{2}$, (b) PProDOT-(EtHx) $)_{2}$ and (c) PMAS.
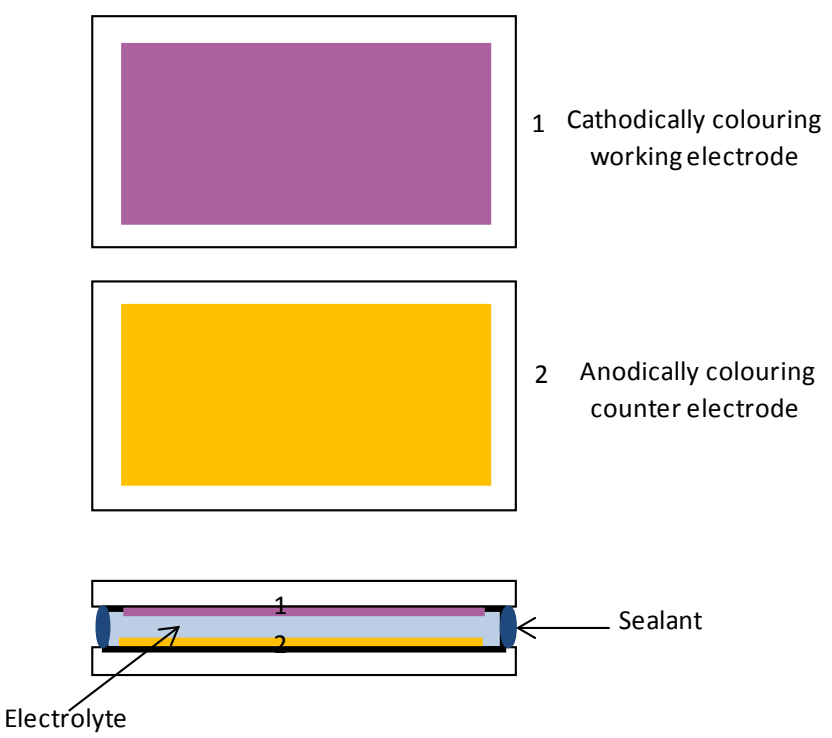

Fig. 2 Demonstration of complimentary ECD.

cross flow dialysis and characterized by the GPC method using 60 polystyrene sulfonate relative standards and found to have Mn 10,510, Mw 15,794 and PDI 1.5.

\subsection{Device fabrication}

\subsubsection{Spin coated PProDOT and PMAS electrode}

PProDOT-(Hx) $)_{2}$ PProDOT-(EtHx $)_{2}$ and PProDOT-Hx $)_{2} /$ 65 (PProDOT-(EtHx) $)_{2}$ mixed polymers at different rations (1:2, 1:1 and 2:1) were all dissolved into a toluene-chloroform mixed were spun onto ITO coated glass at $800 \mathrm{rpm}$ with an acceleration speed of $550 \mathrm{rpm} / \mathrm{s}$ for $15 \mathrm{~s}$. The coated substrates were then dried on hot plate at $50{ }^{\circ} \mathrm{C}$ for 5 minutes. ITO glass substrates were 70 sourced from Zhuhai Kaivo Electronic Components Co., Ltd, China that have a resistivity of $15 \Omega / \square$ and a glass thickness of $1.1 \mathrm{~mm}$. Before coating, the ITO glass was cleaned with detergent in an ultrasonication bath, and then rinsed with isopropanol and acetone before use.

75 PMAS films were also obtained by spin coating technique from PMAS aqueous solution. PMAS was dissolved in deionised water at $60 \mathrm{mg} / \mathrm{mL}$ and spin coated on ITO glass at $3500 \mathrm{rpm}$ with an accelerating speed of $1760 \mathrm{rpm} / \mathrm{s}$ and subsequently dried on a hot plate at $50{ }^{\circ} \mathrm{C}$ for 5 minutes. ITO glass used in this step was pre80 treated as discussed above,then followed by $30 \mathrm{~s}$ plasma treatment to improve the hydrophilic property of the substrate surface. 

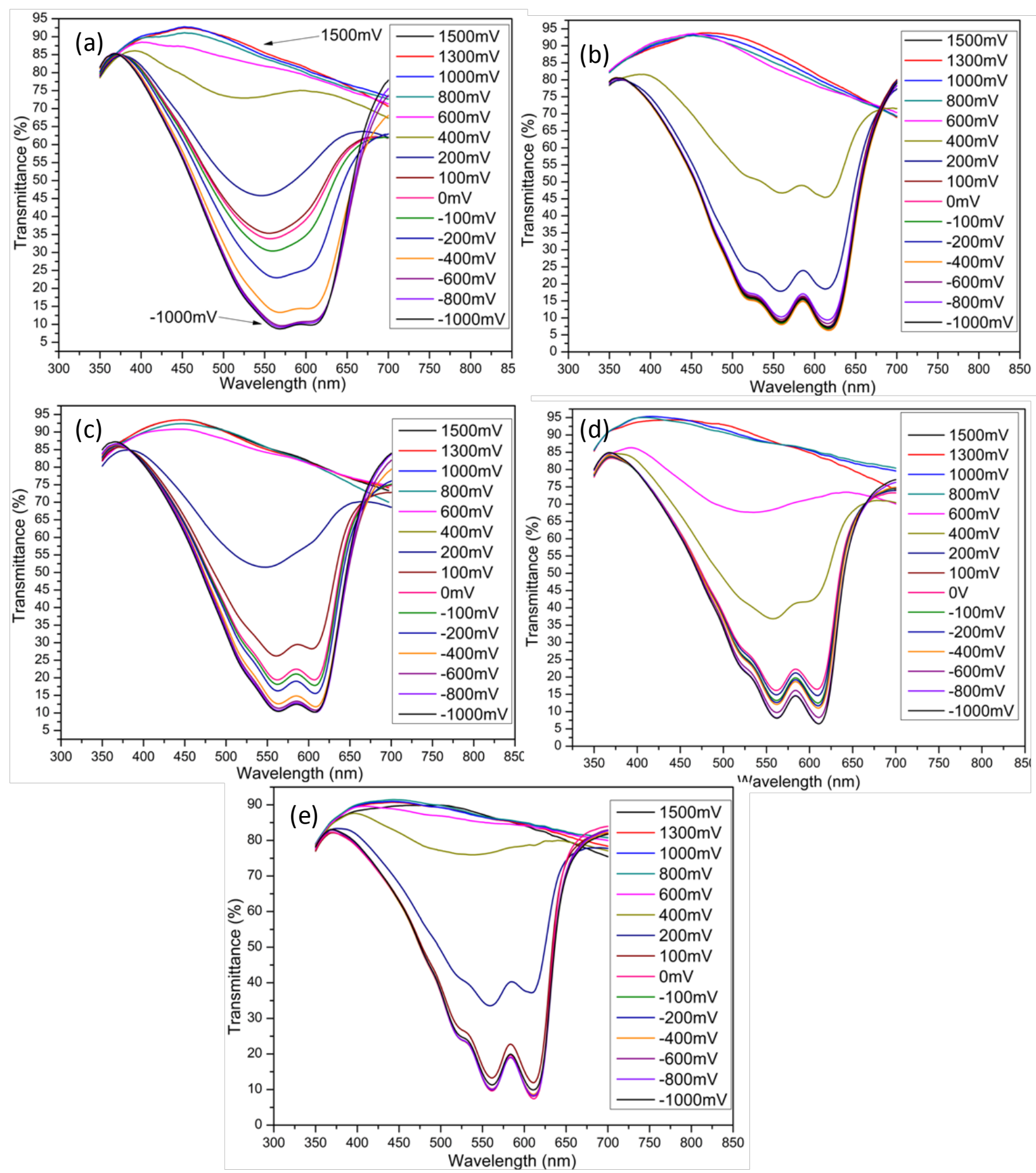

Fig. 3 Spectroelectrochemistry of spin coated slides of (a) PProDOT-(Hx) $)_{2}$; (b) PProDOT-(EtHx $)_{2}$; (c) PProDOT-(Hx) $)_{2}$ : PProDOT-(EtHx $)_{2}=2: 1$; $($ d) PProDOT-(Hx) $)_{2}$ : PProDOT-(EtHx $)_{2}=1: 1$; and (e) PProDOT-(Hx) $)_{2}$ :PProDOT-(EtHx $)_{2}=1: 2$ in EMI TFSI

\subsubsection{Assembly of ECDs}

$55 \mathrm{~cm} \times 5 \mathrm{~cm}$ size ITO glass sheets coated with PProDOT and PMAS were assembled as ECDs for electrochemical and colour measurement. The devices were designed and assembled as shown in Figure 2. Two slides were separated by $100 \mu \mathrm{m}$ spacer and sealed by UV and visible light curing glass bonding 10 adhesives (Dymax 425) over the edge. A 1-2 mm opening was left to allow the entry of electrolyte. After vacuum filling hollow EC cell with an ionic liquid (IL) electrolyte, the opening was sealed and ECDs were ready for characterization.

2.3 Electrochemical, spectroelectrochemical, physical and 15 colour measurement

Electrochemical characterisation was carried out using a VoltaLab 80 potentiostat (PGZ402 with VoltaMaster 4 software). For the ECD experiments, the potentiostat working electrode leads were connected to the PMAS coated ITO glass electrode, 20 with the counter and reference electrodes leads shorted together and connected to PProDOT coated electrode. In situ visible region spectra were recorded in transmission mode using an 
Table 1 Spectroelectrochemistry transmittance of PProDOT and PMAS coated slides. The transmittance numbers were read at $+1.5 \mathrm{~V}$ and $-1.0 \mathrm{~V}$ and the difference between them $(\Delta \mathrm{T})$ at wavelength of $550 \mathrm{~nm}$. The electrodes were cycled in EMI TFSI.

\begin{tabular}{cccc}
\hline & $\begin{array}{c}\text { Transmittance } \\
\text { at }+1.5 \mathrm{~V}(\%)\end{array}$ & $\begin{array}{c}\text { Transmittance } \\
\text { at }-1.0 \mathrm{~V}(\%)\end{array}$ & $\Delta \mathrm{T}(\%)$ \\
\hline PProDOT-(Hx) $)_{2}$ & 84.97 & 9.77 & 75.2 \\
PProDOT $(\mathrm{EtHx})_{2}$ & 87.80 & 15.55 & 72.25 \\
Hx: $\mathrm{Et}=2: 1$ & 84.99 & 9.96 & 75.03 \\
Hx: $\mathrm{Et}=1: 1$ & 85.33 & 9.30 & 76.03 \\
Hx: $\mathrm{Et}=1: 2$ & 85.37 & 9.82 & 75.55 \\
\hline
\end{tabular}

5

Agilent UV 8453 spectrophotometer. The morphologies and thicknesses of spun films were recorded by optical profilometryVeecoWyko NT9100 before device fabrication. Colour measurement and transmittance measurement ( $\mathrm{Y}$ values in ${ }_{10}$ CIE 1931 colour system) of coated slides was carried out using a ColourQuest XE colour measurement spectrometer (Hunterlab, USA) analysis system at 550nm. The photopic contrast (differences of $\mathrm{Y}$ value between dark state and bleached state) of ECDs were characterized by the $\mathrm{Y}$ value (luminance) of CIE 151931 colour system and the colour of dark-state ECDs were characterized and described in the form of CIE L*a*b* coordinates, a uniform colour space defined by CIE in 1976.

\section{Results and discussion}

\subsection{Physical characterization of spin coated films}

20 Prior to device assembly, surface profilometry images of PProDOT and PMAS films (Figure S1 (a), (b)) illustrated that smooth and uniform films were obtained by spin coating. Film thicknesses of spun films were calculated from profile curves (Figure S1 (c), (d)) and listed in Table 1. Results showed that

${ }_{25}$ PProDOT-(Hx) $)_{2}$ films had a thickness of $\sim 120 \mathrm{~nm}$ while the thickness value of PMAS films were $\sim 60 \mathrm{~nm}$. Transmittances (Y values in CIE 1931 colour system) of the slides were also characterized using the Hunter Lab. The transmittances of the as spun PProDOT coated slides ranged from $17.8 \%$ to $18.9 \%$ while 30 those of PMAS electrodes located in $78 \%$ to $79 \%$ (Table S1). The as-spun PProDOT-(Hx) $)_{2}$ had a distinctive purple-red colour while the PProDOT-(EtHx) $)_{2}$ coated slides were dark blue. In the asprepared state the colour of the mixed PProDOT polymer coated slides were closer in clour to the PProDOT- $(\mathrm{Hx})_{2}$.

\section{3.2 Spectroelectrochemistry}

Spectroelectrochemical characterization was carried out on individually coated PProDOT slides to determine the switching characteristics of the electrodes. PProDOT-(Hx) $)_{2}$, PProDOT$(\text { EtHx })_{2}$ and PProDOT-(Hx) 2 /PProDOT-(EtHx $)_{2}$ mixed polymers 40 at different ratios (1: 2, 1: 1, 2: 1) were spin coated on $5 \mathrm{~cm} \mathrm{x} 5 \mathrm{~cm}$ ITO coated glass as described in 2.2.1. After drying on hot plate, the slides were cut carefully into 1 x $5 \mathrm{~cm}$ electrodes for spectroelectrochemical characterization in an liquid electrolyte, 1Ethyl-3-methyl imidazolium Trifluoromethanesulfonate (EMI45 TFSI). Asymmetric square-wave potentials were applied to the coated PProDOT electrode, starting at the open circuit potential, $0 \mathrm{~V}$, and extending in both the positive and negative directions. The switching potential limits were $-1.0 \mathrm{~V}$ for dark-state and + $1.5 \mathrm{~V}$ for bleached state, representing a compromise between so enhancing the switching kinetics, and avoiding oxidation/reduction damage of the electrochromic materials.

For each PProDOT coating type, measurement of spectral data was repeated three times and found to be coincident for each coating type. Spectroelectrochemical responses from 350-700 nm 55 (Figure 3) showed that the peak shape of PProDOT-(Hx) $)_{2}$ and PProDOT-(EtHx $)_{2}$ were different from each other. The peaks of PProDOT-(EtHx) $)_{2}$ UV-vis spectrum $(-1.0 \mathrm{~V})$ at 550nm and 635 $\mathrm{nm}$ are shaper than those corresponding peaks of PProDOT-(Hx) $)_{2}$ UV-vis spectrum. In addition, PProDOT-(EtHx) $)_{2}$ slides could be 60 fully switched at a lower potential $(200 \mathrm{mV})$ than the PProDOT$(\mathrm{Hx})_{2}(-600 \mathrm{mV})$.

The observed spectral peak shapes of PProDOT polymer mixtures shifted from PProDOT-(Hx) $)_{2}$ towards the PProDOT$(\mathrm{EtHx})_{2}$ with the increasing loading of PProDOT-(EtHx $)_{2}$ in ${ }_{65}$ polymer composites. This approach illustrated that the dark-state colours of spun electrodes changed significantly with polymer components, providing a degree of colour tunablility for the ECD. The observed transmittance difference at $550 \mathrm{~nm}$ between dark and bleached states was $75.2 \%$ for PProDOT-(Hx) $)_{2}$ and 75 70 to $76 \%$ for the mixed PProDOT-(Hx) $)_{2} /$ PProDOT-(EtHx) $)_{2}$ electrochromes (Table 1). Both of these systems had higher transmittance switching ranges than the PProDOT-(EtHx) $72.3 \%$ ). Based on these single electrode transmittance switching ranges is was evident that assembled ECDs based on PProDOT$75(\mathrm{Hx})_{2}$ or mixed polymers coated slides with PMAS complementary electrodes should result in higher photopic contrast than ECDs assembled with PProDOT-(EtHx) $)_{2}$ coated slides.

\subsection{Colour measurement of ECDs}

80 In order to study the influence of mixed polymers on the ECDs dark-state colours and qualify the assumption of improved photopic contrast, five ECDs were fabricated with PMAS coated slides and PProDOT coated slides (PProDOT-(Hx) 2 , PProDOTEtHx $_{2}$, PProDOT-(Hx) $)_{2}$ : PProDOT-(EtHx $)_{2}=1: 2,1: 1$ and 2:1) 85 and filled with EMI TFSI. A device switching potential window from $-1.3 \mathrm{~V}$ and $0.8 \mathrm{~V}$ was selected to bleached and darken the ECD based upon the above single electrode spectroelectrochemistry studies (Figure 3) while accounting for the need to charge balance the redox processes associated with ${ }_{90}$ PProDOT and the PMAS counter electrode. The switching potentials were applied for a total of 10 seconds in the ECD to permit full colouration before taking measurements measurements. Dark $(0.8 \mathrm{~V})$ and bleached state $(-1.3 \mathrm{~V})$ colours were measured at the end of each switching period. The CIE ${ }_{95} \mathrm{~L} * \mathrm{a} * \mathrm{~b} *$ (CIELAB) colour space system, adopted by the International Commission on Illumination (CIE), was used to express colour measurement results in this work. CIELAB is a uniform colour space defined to be a geometrical construct containing all possible colours, and is a standard commonly used 100 in the paint, plastic and textile industries. In the $\mathrm{L}^{*} \mathrm{a} \mathrm{b}^{*}$ chromaticity diagram, $\mathrm{L}^{*}$ defines lightness $(0=$ black and $00=$ diffuse white) while a $+a^{*}$ relates to the red direction, $-a * i s$ the green direction, $+b^{*}$ is the yellow direction, and $-b^{*}$ is the blue direction. The center of the chromaticity diagram $(0,0)$ is 105 achromatic; as the values of $\mathrm{a}^{*}$ and $\mathrm{b}^{*}$ increase, the saturation of the colour increases.

It has been reported that complementary ECDs with two 
dissimilar electrochromic layers, each with its individual
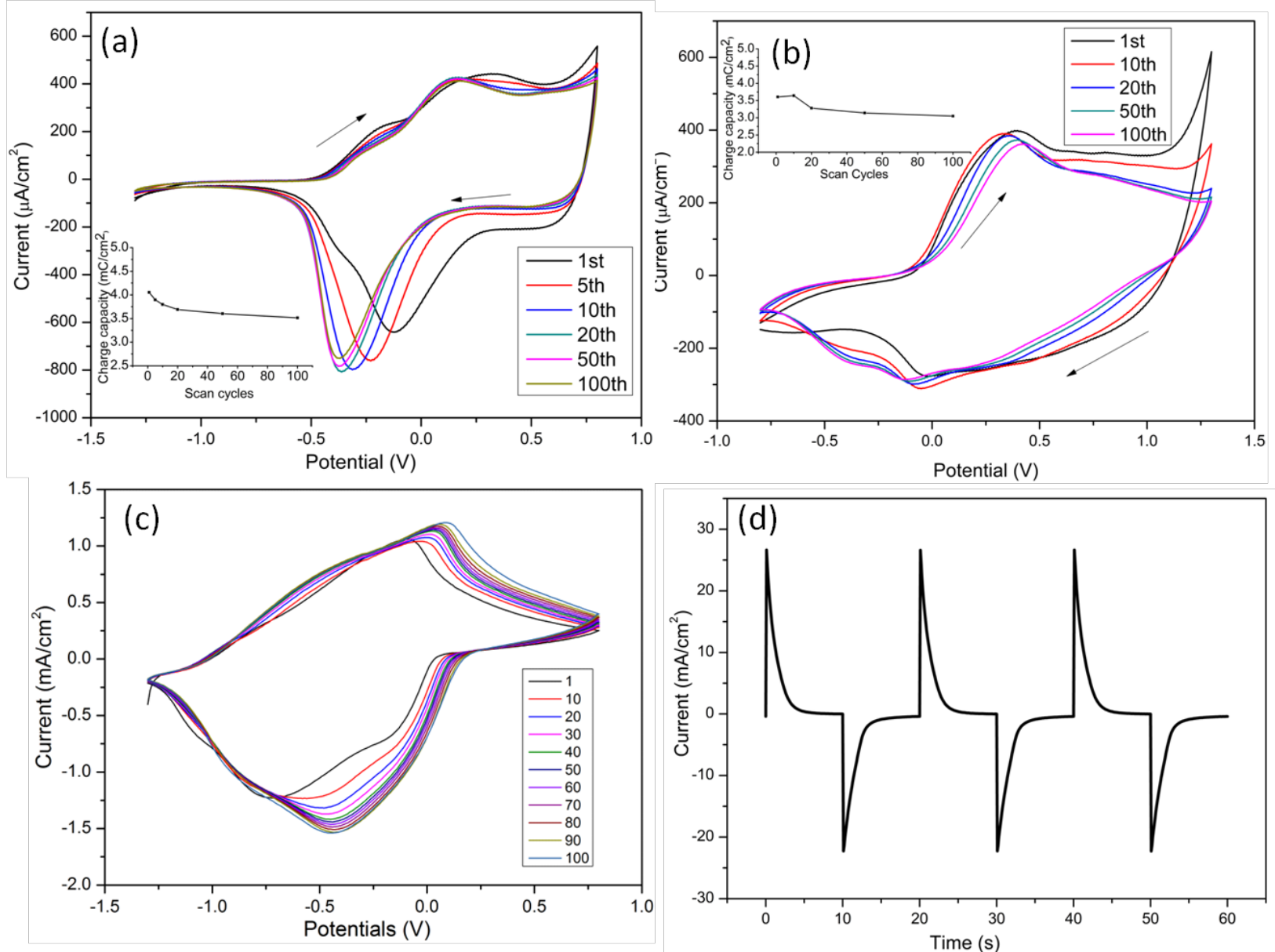

Fig. 4 (a) CV of spun PMAS. Scan range: -1.3V to 0.8V; (b) CV of spun PProDOT(1:1 ratio). Scan range: -0.8V to 1.3V; (c) CV and (d) Amprometry of ECD made from PMAS and PProdot (1:1 ratio).CV scan range: $-1.3 \mathrm{~V}$ to $0.8 \mathrm{~V}$, Switching potentials: $-1.3 \mathrm{~V}$ and $0.8 \mathrm{~V}$. Scan speed: $100 \mathrm{mV} / \mathrm{s}$. Electrolyte: EMI-TFSI ion liquid.

Table 2 The relationship between the transmittances of spun films and charge capacities and ECD performance based on these spun films.

\begin{tabular}{lllllllr}
\hline $\begin{array}{l}\text { Transmittance } \\
\text { of PMAS films } \\
(\mathrm{T} \%)\end{array}$ & $\begin{array}{l}\text { Charge } \\
\text { capacity } \\
\left(\mathrm{mC} / \mathrm{cm}^{2}\right)\end{array}$ & $\begin{array}{l}\text { Transmittance } \\
\text { of PProDOT } \\
\text { films (T\%) }\end{array}$ & $\begin{array}{l}\text { Charge } \\
\text { capacity } \\
\left(\mathrm{mC} / \mathrm{cm}^{2}\right)\end{array}$ & $\begin{array}{l}\text { ECD made from spun Charge } \\
\text { PMAS and PProDOT capacity } \\
\text { films with different } \\
\text { thinknesses } \\
\text { ratio }\end{array}$ & $\begin{array}{l}\text { Upper } \\
\text { transmittance } \\
\text { of ECD(T\%) }\end{array}$ & $\begin{array}{l}\text { Lower } \\
\text { transmittance } \\
\text { of ECD(T\%) }\end{array}$ & $\begin{array}{l}\text { Contrast } \\
(\%)\end{array}$ \\
\hline 73.64 & 3.68 & 14.24 & 3.93 & $\begin{array}{l}\text { PMAS 77.86\%, } \\
\text { PProDOT 18.06\% }\end{array}$ & 0.97 & 69.14 & 11.32 \\
\hline 77.86 & 3.01 & 18.06 & 3.09 & $\begin{array}{l}\text { PMAS 73.64\%, } \\
\text { PProDOT 23.67\% }\end{array}$ & 1.78 & 58.32 & 15.47 \\
\hline 81.37 & 2.64 & 23.67 & 2.06 & $\begin{array}{l}\text { PMAS 81.37\%, } \\
\text { PProDOT 14.24\% }\end{array}$ & 0.67 & 44.15 & 9.68 \\
\hline
\end{tabular}

thickness and charge storage capacities, must be matched (or charge balanced) in order to reach a maximum photopic contrast $^{38,39}$. In these reports ${ }^{38,39}$ the charge capacities of the 10 electrodes were controlled by applying defined amount of charge during the electrochemical polymerization of the electrochromic film layers. In this work, we optimized the spin coating conditions to control the amount of material deposited onto ITO glass electrodes.

15 The cyclic voltammetry (CV) of spun PMAS and PProDOT (PProDOT- $(\mathrm{Hx})_{2}$ : PProDOT-(EtHx $\left.)_{2}=1: 1\right)$ films with different thicknesses were characterized to calculate charge capacities via the equation:

\section{$Q=A / 2 f$}

${ }_{20}$ Where $\mathrm{A}$ is integral area of the cyclic voltammogram loop, $\mathrm{f}$ is the scan rate. A device switching potential window from $-1.3 \mathrm{~V}$ and $0.8 \mathrm{~V}$ was selected to bleached and darken the ECD based upon the single spectroelectrochemistry results (Figure 3). Consequently the voltage window for $\mathrm{CV}$ studies was selected to 25 be $-1.3 \mathrm{~V}$ to $+0.8 \mathrm{~V}$ for PMAS and $-0.8 \mathrm{~V}$ to $+1.3 \mathrm{~V}$ for PProDOT. The CV measurements were made using a three-electrode system in EMI-TFSI ion liquid with $\mathrm{Ag} / \mathrm{AgCl}$ reference electrode at $100 \mathrm{mV} / \mathrm{s}$ for 100 cycles.

From Figure 4 (a), the reduction peak current increased with scan 30 cycle and shifted to a lower voltage over initial 20 cycles, 


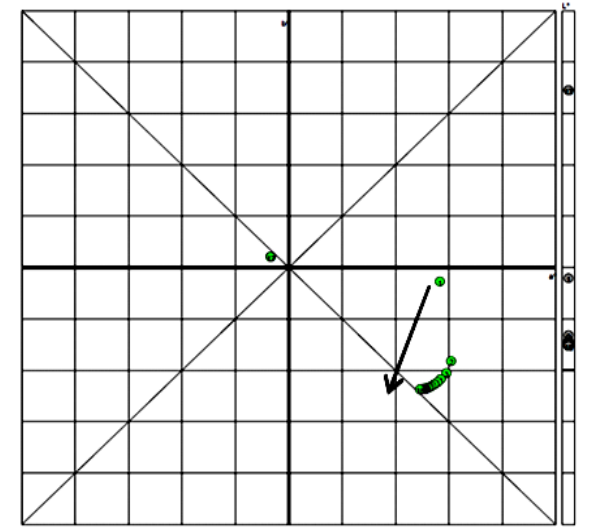

(a)

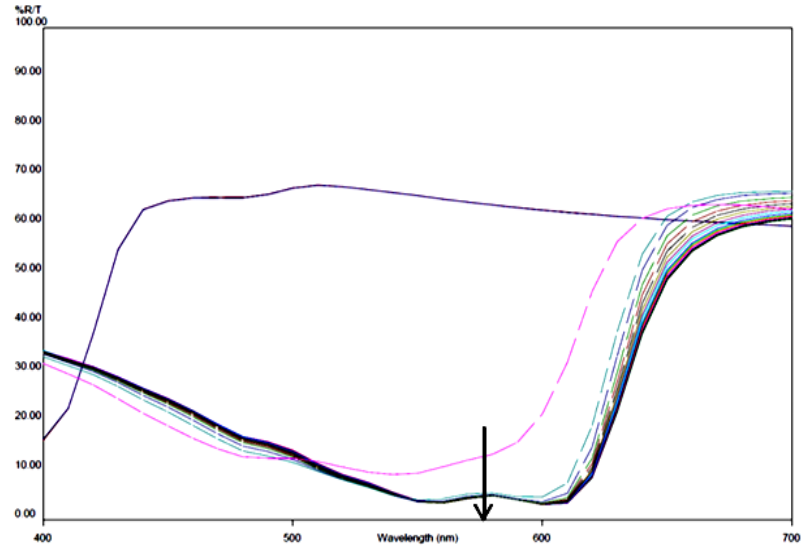

(b)

Fig. 5 (a) Colour changes and (b) Uv-vis spectrum of PProDOT-(Hx) $)_{2}$ PProDOT-(EtHx)2 = 1: 1 ECD in the first 100 cycles. Switching potentials: $-1.3 \mathrm{~V}$ and $0.8 \mathrm{~V}$.

Table 3 Dark-state L*a*b* values and contrasts of PProDOT-(Hx) 2 , PProDOT-(EtHx)2 and mixed polymers ECDs

\begin{tabular}{|c|c|c|c|c|c|c|}
\hline$\overline{\text { ECDs }}$ & L*a*b* values & $\begin{array}{l}\text { Transmittance } \\
\text { of PProDOT } \\
\text { slide (T\%) }\end{array}$ & $\begin{array}{c}\text { Transmittance } \\
\text { of PAMS } \\
\text { slide(T\%) }\end{array}$ & $\begin{array}{c}\text { Upper } \\
\text { transmittance } \\
\text { of ECD (T\%) }\end{array}$ & $\begin{array}{c}\text { Lower } \\
\text { transmittanc } \\
\text { e of ECD } \\
(\mathrm{T} \%) \\
\end{array}$ & $\begin{array}{c}\text { Photopic contrast }=\text { Upper } \\
\text { ECD transmittance - } \\
\text { Lower ECD transmittance } \\
(\%)\end{array}$ \\
\hline$\overline{\text { PProDOT(EtHx })_{2}}$ & $\begin{array}{l}L^{*}=50.43 ; a^{*}=13.4 \\
b^{*}=-13.87 ; \text { Colour: }\end{array}$ & 18.86 & 78.74 & 69.79 & 18.78 & 51.17 \\
\hline PProDOT(Hx) $)_{2}$ & $\begin{array}{l}L^{*}=38.39 ; a^{*}=37.11 ; \\
b^{*}=-28.19 ; \text { Colour: }\end{array}$ & 18.06 & 78.25 & 64.08 & 10.89 & 57.19 \\
\hline $\mathrm{Hx}: \mathrm{Et}=2: 1$ & $\begin{array}{l}\mathrm{L}^{*}=39 ; \mathrm{a}^{*}=36.27 \\
\mathrm{~b}^{*}=-28.48 ; \text { Colour: }\end{array}$ & 18.2 & 78.6 & 69.05 & 12.63 & 56.72 \\
\hline $\mathrm{Hx}: \mathrm{Et}=1: 1$ & $\begin{array}{l}\mathrm{L}^{*}=39.09 ; \mathrm{a}^{*}=30.14 \\
\mathrm{~b}^{*}=-31.62 ; \text { Colour: }\end{array}$ & 17.88 & 77.41 & 68.85 & 10.14 & 58.71 \\
\hline $\mathrm{Hx}: \mathrm{Et}=1: 2$ & $\begin{array}{l}\mathrm{L}^{*}=42.21 ; \mathrm{a} *=25.62 \\
\mathrm{~b}^{*}=-29.38 ; \text { Colour: }\end{array}$ & 18.09 & 78.46 & 67.38 & 10.66 & 56.42 \\
\hline
\end{tabular}

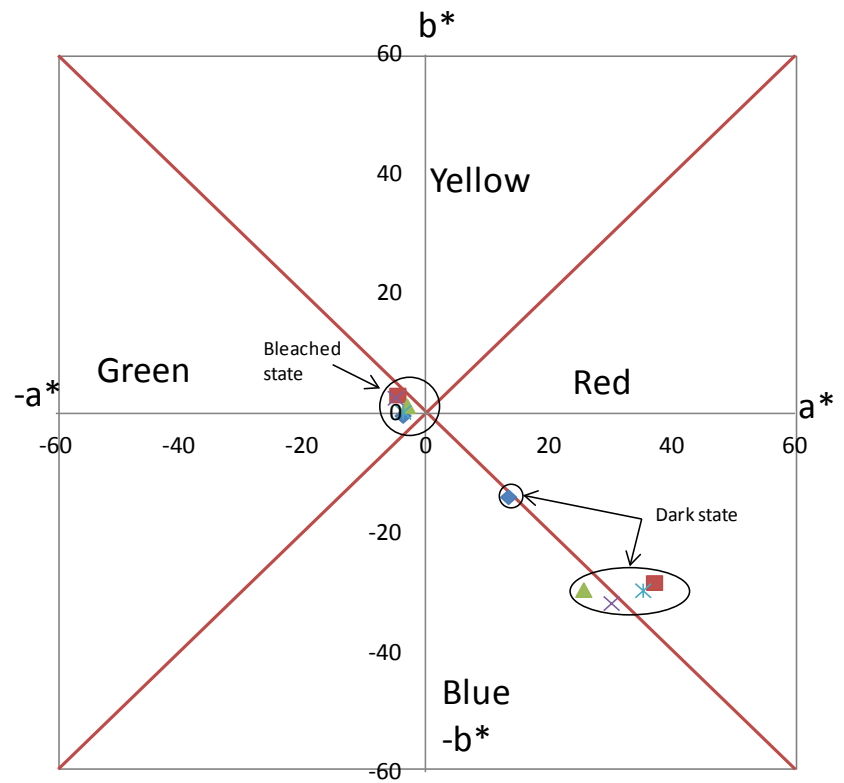

5 PProDOT-EtHx2 $\square$ PProDOT-Hx2 $\triangle \mathrm{Hx}: \mathrm{Et}=1: 2 \quad \times \mathrm{Hx}: \mathrm{Et}=1: 1 \quad * \mathrm{Hx}: \mathrm{Et}=2: 1$

Fig. 6 Data plot of dark and bleached state colours of PProDOT-(Hx) PProDOT-(EtHx)2 and mixed polymers ECDs in CIELAB colour space.

where thereafter it was observed to remain stable. The charge capacity calculated from integral area of CV decreased gradually
10 until it stabilised after 20 cycles (inset graph in Figure 4 (a)). PProDOT presented similar behavior in comparison with PMAS, where the current at $+1.3 \mathrm{~V}$ decreased over 20 scan cycles and remained relatively stable thereafter (Figure 4 (b)). This behaviour explains why the response current in the assembled 15 ECD CV was more significant within first 20 cycles (Figure 4 (c)).

The influence of charge balance between PMAS and PProDOT on ECD performance was evaluated by assembling devices with different charge ratios (Table 3). The charge capacity values were 20 calculated from the $100^{\text {th }}$ cycle of the CV curves for balance consideration. Three charge ratios, $0.97,1.78,0.67$, were adopted for device performance characterization with the highest photopic contrast being obtained when the charge ratio was close to 1 . PMAS (charge ratio $=1.78$ ) or PProDOT (Charge ratio $=0.67$ ) 25 films were not fully switched when in the bleached state, affecting the upper transmittance values of ECD and thereby reducing ECD contrast. Chronoamperometry of ECD with 0.97 charge ratio (Figure 4 (d)) confirmed the charge balance status of the device from another aspect as response current came back to 30 zero at the end of switch intervals.

Initial studies on dark-state colours $(+0.8 \mathrm{~V})$ were carried out and a representative response is shown for a 1:1 mixed polymer ECD (Figure 5). This ECD illustrated a clear device "warm-up" period, in which the dark-state colours of mixed polymer ECD kept 


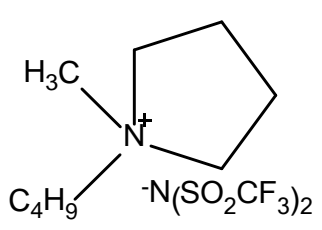

(a)<smiles>CCCCCC[N+]1(C)CCCC1</smiles>

(b)<smiles></smiles>

(c)<smiles></smiles>

(d)

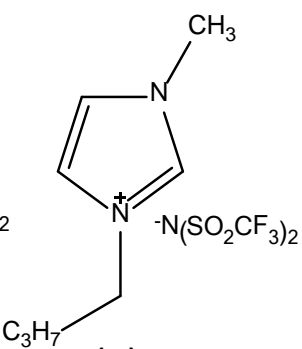

(e)

Fig. 7 Chemical structures of ionic liquid electrolytes (a) 1-Butyl-1-Methylpyrrolidinium-Bis(trifluromethanesulfonyl)imide (P1,4-TFSI), (b) 1-Propyl-1Methylpyrrolidinium-Bis(trifluromethanesulfonyl)imide(P1,3-TFSI), (c) 1-Ethyl-3-Methylimidazolium dicynamide (EMI-DCA), (d) 1-Ethyl-3Methylimidazolium-Bis(trifluromethanesulfonyl)imide (EMI-TFSI) and (e) 1-Butyl-3-Methylimidazolium-Bis(triffluromethanesulfonyl)imide (BMITFSI).

Table 4 Dark-state $\mathrm{L}^{*} \mathrm{a}{ }^{*}{ }^{*}$ values and contrasts of PProDOT-(Hx) $)_{2}$ : PProDOT-(EtHx $)_{2}=1: 1$ ECDs filled with different ionic liquids.

\begin{tabular}{|c|c|c|c|c|c|c|}
\hline Electrolyte & L*a*b* values & $\begin{array}{l}\text { Transmittance } \\
\text { of PProDOT } \\
\text { slide (T\%) } \\
\end{array}$ & $\begin{array}{c}\text { Transmittance } \\
\text { of PAMS } \\
\text { slide(T\%) } \\
\end{array}$ & $\begin{array}{c}\text { Upper } \\
\text { transmittance } \\
\text { of } \mathrm{ECD}(\mathrm{T} \%) \\
\end{array}$ & $\begin{array}{c}\text { Lower } \\
\text { transmittance } \\
\text { of } \operatorname{ECD~}(\mathrm{T} \%) \\
\end{array}$ & $\begin{array}{l}\text { Photopic contrast = Upper } \\
\text { ECD transmittance - Lower } \\
\text { ECD transmittance (\%) }\end{array}$ \\
\hline EMI-DCA & $\begin{array}{l}L^{*}=46.54 ; a^{*}=36.54 \\
b^{*}=-18.25 ; \text { Colour: }\end{array}$ & 18.11 & 78.06 & 43.22 & 26.38 & 16.84 \\
\hline P14 -TFSI & $\begin{array}{l}L^{*}=38.58 ; a^{*}=34.42 \\
b^{*}=-29.89 ; \text { Colour: }\end{array}$ & 17.95 & 77.93 & 66.2 & 10.52 & 55.68 \\
\hline P13 -TFSI & $\begin{array}{l}L^{*}=39.23 ; a^{*}=33.22 \\
b^{*}=-28.24 ; \text { Colour: }\end{array}$ & 18.33 & 78.65 & 67.78 & 11.29 & 56.49 \\
\hline BMI -TFSI & $\begin{array}{l}L^{*}=39.39 ; a^{*}=28.55 \\
b^{*}=-31.76 ; \text { Colour: }\end{array}$ & 18.25 & 78.61 & 68.29 & 10.96 & 57.33 \\
\hline
\end{tabular}

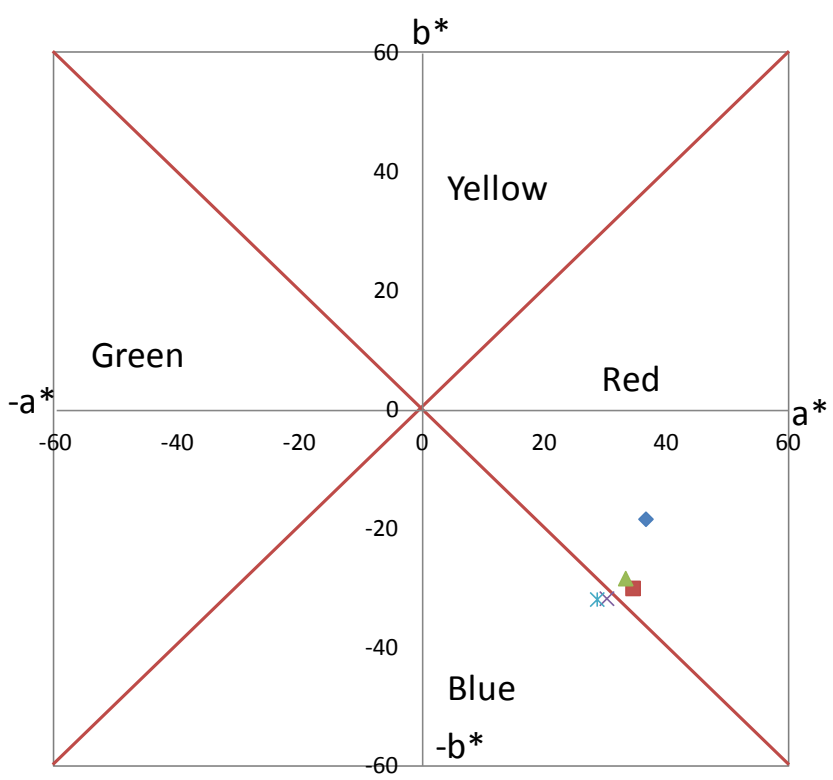

$\triangle$ EMI-DCA P14-TFSI AP13-TFSI ×EMI-TFSI *BMI-TFSI

Fig. 8 Data plot of dark-state colours of PProDOT-(Hx)2: PProDOT-

$($ EtHx)2 = 1: 1 ECDs filled with different ionic liquids.

improving until a balance was reached, presumably due to polymer and dopant rearrangements in the ionic liquid electrolyte environment. Colours and contrast transmittances for this device

15 were recorded at the end of each potential switching window at either $-1.3 \mathrm{~V}$ or $0.8 \mathrm{~V}$ in 10 second intervals. The dark-state colour of this device was noted to change with switching cycles, with $+a^{*}$ decreased with a concomitant increase in $-b^{*}$. In general, the ECD shifted from the red region to blue region over the warm-up 20 period (Figure 5 (a)). Interestingly, the ECD photopic contrast didn't change significantly during this warm up period, fluctuating only slightly from 58 to 59\%. The same cycling experiments were carried out again on the second, fourth, seventhdays and it was determined that the device performance as 25 coincident with final charge balanced ECD performance achieved after the initial device warm-up. This suggests that that the warmup phase was a one-time process that arises from equilibration interaction of ionic liquid electrolyte with cathodically and anodically colouring polymer doping processes. This 30 phenomenon has been reported previously for PEDOT:PSS ${ }^{32}$. Similar ECD warm-up behavior was observed for all other electrochromic systems investigated. For this reason 150 switching cycles were carried out on all ECDs systems before measuring the devices dark-state colours and contrasts to ensure 35 all the devices reaching a balanced, , Table 3 and Figure. 6. The bleached state colours of all five devices did not show much variation and were nearly colourless, located near $(0,0)$ origin, Figure 6. Contrasting this, the dark-state ECD colours changed significantly with the composition of cathodically colouring 40 electrochromic layer. Dark-state colours of ECDs made with PProDOT-(EtHx $)_{2}$ and mixed polymers PProDOT-(Hx) $)_{2}$ : PProDOT-(EtHx $)_{2}=1: 2,1: 1$ sit within the blue region of CIELAB colour space while PProDOT-(Hx) $)_{2}$ and PProDOT$(\mathrm{Hx})_{2}$ : PProDOT-(EtHx $)_{2}=2: 1$ ECDs showed deep purple red 
colours, which were located within red region of CIELAB colour space. For the three mixed PProDOT polymers ECDs investigated the dark-state colour moved gradually from red region to blue region with an increase of the PProDOT-(EtHx $)_{2}$

5 weight ratio within mixed coating. In addition, the absolute values of dark-state $\mathrm{a}^{*}$ and $\mathrm{b}^{*}$ values for the PProDOT-(Hx) ECD and mixed polymers ECDs were higher than the ECD made with pure PProDOT-(EtHx) $)_{2}$ coated slide. This illustrated PProDOT-(Hx) $)_{2}$ possessed higher colour saturation than ${ }_{10}$ PProDOT-(EtHx $)_{2}$ and made great contribution in mixed polymer system. The incorporation of PProDOT-(EtHx) $)_{2}$ into mixed polymer changed ECDs' dark-state colours without significantly reducing colour saturation. The results of device contrasts studies (Table 3) agreed well with spectroelectrochemistry 15 investigations. The contrast of PProDOT-(EtHx) $)_{2}$ ECD (51.17\%) was the lowest of the five systems studied while the contrast of all other ECDs achieved over 55\%, demonstrating PProDOT$(\mathrm{Hx})_{2}$ domain not only dominated spectroelectrochemical performance of the device influencing both colour saturation and 20 device contrast in mixed PProDOT ECDs. Significantly a more esthetically pleasing dark blue coloured ECD with higher contrast and colour saturation can be achieved using mixed polymers coated PProDOT slides (Hx: Et $=1: 2$ or $1: 1$ ) than individual PProDOT layers themselves.

\section{3.4 Influence of Electrolyte}

Electrolyte type plays a significant role in ECD performance, transferring charges, changing the doping state and influencing the conformation of polymer chains $\frac{32}{2}$. To probe the influence of this critical parameter, four hydrophilic ionic liquid (IL) 30 electrolytes, EMI-TFSI, 1-Butyl-3-MethylimidazoliumBis(triffluromethanesulfonyl)imide (BMI-TFSI), 1-Butyl-1Methylpyrrolidinium-Bis(trifluromethanesulfonyl)imide (P14TFSI), 1-Propyl-1-MethylpyrrolidiniumBis(trifluromethanesulfonyl)imide (P13-TFSI) and one 35 hydrophobic liquid electrolyte 1-Ethyl-3-Methylimidazolium dicynamide (EMI-DCA) (Figure 7) were selected to explore the influence of working electrolytes on the contrast and colour changes. All the experiments were carried out with ECDs made with PProDOT-(Hx) $)_{2}$ : PProDOT-(EtHx $)_{2}=1: 1$ mixed polymer 40 coated PProDOT slides. The test conditions were the same as those used in Section 3.3 following 150 warm-up cycles.

When the hydrophilic EMI-DCA was used as an electrolyte a sharp reduction in the contrast range of the ECD was noted, Table 4 and Figure 8. In this electrolyte the ECD could not be fully 45 switched into the colourless state at $-1.3 \mathrm{~V}$. ECDs filled with IL's containing the same anion but with different cations had similar photopic contrast ranging from 57 to 59\%. However, the balanced dark-state colours were clearly influenced by the type of ionic liquid. P13-TFSI and P14-TFSI, which had similar cationic 50 ion structure, created deep purple dark-state colours located in red region of CIELAB colour space while BMI-TFSI gave a similar performance in dark-state colour in comparison to EMI-TFSI with only a slight difference observed for the colour lightness and saturation where the BMI-TFSI filled ECD had lower $a^{*} b^{*}$ 55 values and colour saturation than EMI-TFSI filled ECD. Significantly, both of these samples expressed a more esthetically blue colour than P13- or P14-TFSI which had a more purple hue.

\subsection{Stability}

ECD stability is one of the most important factors that determine 60 the life of devices. Stability can refer to both long term materials storage stability and long-term ECD cycle-life stability. Ideally, ECDs should be stable after long term inactive storage and not display any significant performance losses. Long-term running stability means ECD should maintain most of its contrast after 65 many thousands of switching cycles. Long term studies on these devices using EMI TFSI have shown that the ECD's keep over $97 \%$ of their original contrast range after six months storage in the open air. Cycle life tests indicated that the PProDOT-(Hx) ECD and mixed PProDOT polymer ECDs had longer cycle life 70 than the PProDOT-(EtHx)2 ECD. Cycle life switching was performed on each device construct over a total of 10,000 cycles to investigate the contrast degeneration. PProDOT-(Hx) $)_{2}$ ECD and mixed polymer ECDs maintained $91.7 \%$ to $93.2 \%$ of the original contrast while PProDOT-(EtHx) $)_{2}$ ECD only kept $85.7 \%$ 75 of the original value.

\section{Conclusions}

Colour tunable large scale ECDs were successfully developed and fabricated using complimentary colouring PProDOT and PMAS polymers. Initial spectroelectrochemistry studies ${ }_{80}$ demonstrated the possibility of using mixtures of PProDOT-(Hx) $)_{2}$ and PProDOT-(EtHx) $)_{2}$ to adjust dark-state colour, photopic contrast and colour saturation of the device. A dark-state ECD colouration from red-purple to dark blue can was successfully modified by changing the ratios of mixed PProDOT component 85 as well as by selective use of the type of ionic electrolyte used in the device.

\section{Acknowledgement}

The authors would like to acknowledge Australia Research Council (ARC) and Samvardhana Motherson Reflectec (SMR) 90 Automotive for funding support and Australian National Fabrication Facility (ANFF) for equipment support.

\section{Notes and references}

Intelligent Polymer Research Institute, ARC Centre of Excellence for Electromaterials Science, University of Wollongong, NSW, Australia; E95 mail: innis@uow.edu.au.

† Electronic Supplementary Information (ESI) available: See DOI: $10.1039 / \mathrm{b} 000000 \mathrm{x} /$

1. M. Berggren, D. Nilsson and N. D. Robinson, Nature materials, 2007, 6, 3-5.

2. S. R. Forrest, Organic Electronics, 2003, 4, 45-48.

3. T. W. Kelley, P. F. Baude, C. Gerlach, D. E. Ender, D. Muyres, M. A. Haase, D. E. Vogel and S. D. Theiss, Chemistry of materials, 2004, 16, 4413-4422.

105 4. H. Klauk, Organic Electronics: Materials, Manufacturing, and Applications, Wiley-VCH, 2006.

5. J. Wang, L. Sun, K. Mpoukouvalas, K. Lienkamp, I. Lieberwirth, B. Fassbender, E. Bonaccurso, G. Brunklaus, A. Muehlebach and T. Beierlein, Advanced Materials, 2009, 21, 1137-1141.

1106 . S. Sakamoto, M. Okumura, Z. Zhao and Y. Furukawa, Chemical physics letters, 2005, 412, 395-398.

7. K. M. Lee, C. Y. Hsu, P. Y. Chen, M. Ikegami, T. Miyasaka and K. C. Ho, Physical Chemistry Chemical Physics, 2009, 11, 3375-3379. 
8. K. M. Lee, P. Y. Chen, C. Y. Hsu, J. H. Huang, W. H. Ho, H. C. Chen and K. C. Ho, Journal of Power Sources, 2009, 188, 313-318.

9. P. Y. Chen, C. P. Lee, R. Vittal and K. C. Ho, Journal of Power Sources, 2010, 195, 3933-3938.

5 10. G. Sonmez, H. Meng and F. Wudl, Chemistry of materials, 2004, 16, 574-580.

11. A. A. Argun, P. H. Aubert, B. C. Thompson, I. Schwendeman, C. L. Gaupp, J. Hwang, N. J. Pinto, D. B. Tanner, A. G. MacDiarmid and J. R. Reynolds, Chemistry of materials, 2004, 16, 4401-4412.

10 12. J. H. Huang, C. Y. Hsu, C. W. Hu, C. W. Chu and K. C. Ho, ACS Applied Materials \& Interfaces, 2010, 2, 351-359.

13. M. Miyaji, Google Patents, 1990.

14. D. J. O'farrell and R. J. Gahan, Google Patents, 1995.

15. B. Loiseaux, P. Robin, A. Oppenlander and J. P. Huignard, Google Patents, 1995.

16. H. W. Heuer, R. Wehrmann and S. Kirchmeyer, Advanced Functional Materials, 2002, 12, 89-94.

17. R. J. Mortimer, Chem. Soc. Rev., 1997, 26.

18. R. J. Mortimer and T. S. Varley, Solar Energy Materials and Solar Cells, 2012, 99, 213-220.

19. P. R. Somani and S. Radhakrishnan, Materials Chemistry and Physics, 2003, 77, 117-133.

20. S. H. Baeck, K. S. Choi, T. F. Jaramillo, G. D. Stucky and E. W. McFarland, Advanced Materials, 2003, 15, 1269-1273.

25 21. S. H. Lee, R. Deshpande, P. A. Parilla, K. M. Jones, B. To, A. H. Mahan and A. C. Dillon, Advanced Materials, 2006, 18, 763-766.

22. D. DeLongchamp and P. T. Hammond, Advanced Materials, 2001, 13, 1455-1459.

23. C. A. Cutler, M. Bouguettaya and J. R. Reynolds, Advanced materials, 2002, 14, 684-688.

24. Q. Pei, G. Zuccarello, M. Ahlskog and O. Inganäs, Polymer, 1994, 35, 1347-1351.

25. B. D. Reeves, C. R. G. Grenier, A. A. Argun, A. Cirpan, T. D. McCarley and J. R. Reynolds, Macromolecules, 2004, 37, 75597569.

26. B. C. Thompson, Y. G. Kim, T. D. McCarley and J. R. Reynolds, Journal of the American Chemical Society, 2006, 128, 12714-12725.

27. R. J. Mortimer, K. R. Graham, C. R. G. Grenier and J. R. Reynolds, ACS Applied Materials \& Interfaces, 2009, 1, 2269-2276.

40 28. P. Tehrani, L. O. Hennerdal, A. L. Dyer, J. R. Reynolds and M. Berggren, J. Mater. Chem., 2009, 19, 1799-1802.

29. M. Berggren, D. Nilsson, P. A. Ersman, P. Tehrani and L. O. Hennerdal, Iontronics: Ionic Carriers in Organic Electronic Materials and Devices, 2010, 131.

45 30. A. L. Dyer, C. R. G. Grenier and J. R. Reynolds, Advanced Functional Materials, 2007, 17, 1480-1486.

31. F. Masdarolomoor and G. G. Wallace, Journal of Materials Chemistry, 2007, 17, 4359-4361.

32. O. Winther-Jensen, S. Desai, R. L. Shepherd, P. C. Innis, B. Winther-

50 Jensen, M. Forsyth, G. G. Wallace and D. R. MacFarlane, Electrochemistry Communications, 2010, 12, 1505-1508.

33. R.J. Mortimer, K. R. Graham, C. R. G. Grenier and J. R. Reynolds, Applied Materials and Interface, 2009, 10, 2269-2276.

34. Y. Zou, W. Wu, G. Sang, Y. Yang, Y. Liu, and Y. Li, Macromolecules 2007, 40, 7231-7237.

35. S. A. Sapp, G. A. Sotzing, J. L. Reddinger and J. R. Reynolds, Advance Materials, 1996, 10, 808-811.

36. S. A. Sapp , G.A. Sotzing, and J. R. Reynolds, Chemistry of Materials, 1998, 10, 2101-2108.

60 37. P. Tehrani, L. O. Hennerdal, A. L. Dyer, J. R. Reynolds and M. Berggren, J. Mater. Chem., 2009, 19, 1799-1802.

38. K. C. Ho, Solar Energy Materials \& Solar Cells, 1999, 56, 271-280.

39. K. C. Ho, Electrochimica Acta, 1999, 44, 3227-3235. 